\title{
White blood cell concentration correlates with increased concentrations of IL-1ra and improvement in WOMAC pain scores in an open-label safety study of autologous protein solution
}

William King ${ }^{2 *}\left(\mathbb{D}\right.$, Walter van der Weegen ${ }^{1}$, Rogier Van Drumpt ${ }^{1}$, Hans Soons ${ }^{1}$, Krista Toler ${ }^{2}$ and Jennifer Woodell-May ${ }^{2}$

\begin{abstract}
Background: There has been debate on which blood components should be included in autologous therapies. Autologous Protein Solution (APS) is a unique blood-derived therapy composed of concentrated white blood cells, platelets, and plasma to contain high concentrations of anti-inflammatory cytokines and anabolic growth factors to potentially address osteoarthritis. The primary aim of the exploratory secondary analysis was to identify characteristics of an Autologous Protein Solution (APS) that may correlate with improved Western Ontario and McMaster Universities Osteoarthritis Index (WOMAC) scores and OMERACT-OARSI responder status after treatment of subjects with an intra-articular injection of APS.
\end{abstract}

Methods: Eleven subjects were enrolled in a pilot study of a single intra-articular injection of APS in subjects with knee osteoarthritis. Two APS kits were processed per patient. The output of the first APS kit was injected intra-articularly. White blood cell (WBC) and cytokine concentrations were measured from the output of the second APS kit. WOMAC surveys were completed at baseline and at follow up visits. Linear regression analyses were performed on the blood components of APS with subject outcomes. Anderson-Darling analysis was used to determine whether the cytokine concentrations in whole blood and APS had a normal distribution. Either paired t-test analyses or Wilcoxon signed-rank analyses were performed for normal and non-parametrically distributed data, respectively.

Results: The WBC concentration in APS was significantly $(p<0.05)$ and strongly $\left(R^{2}>0.7\right)$ correlated with IL-1 ra in APS but not significantly correlated with IL-1 $\beta$. The ratio of IL-1ra to IL-1 $\beta$ in APS was significantly correlated with improved WOMAC pain scores one week and six months post-injection. $85.7 \%$ of subjects whose APS had a IL-1 ra:IL-1 $\beta$ ratio greater than 1000 or a WBC count greater than $30 \mathrm{k} /$ I were OMERACT-OARSI responders six months post-injection.

Conclusions: The correlations between the IL-1ra:IL-1 $\beta$ ratio and WBC concentration in a subject's APS and their WOMAC pain scores and classification as OMERACT-OARSI responders suggest the potential utility of these characteristics as diagnostic markers. Additional studies are ongoing to determine whether APS is safe and effective and to further evaluate the relationship between APS composition and clinical outcomes.

Trial Registration: (NCT01773226)

Keywords: Inflammation, Pain, Function, IL-1ra, Growth factor, Platelet-rich plasma

* Correspondence: William.King@biomet.com

${ }^{2}$ Biomet, 56 East Bell Drive, Warsaw, IN 46581, USA

Full list of author information is available at the end of the article

\section{Springer}

(c) 2016 King et al. Open Access This article is distributed under the terms of the Creative Commons Attribution 4.0 International License (http://creativecommons.org/licenses/by/4.0/), which permits unrestricted use, distribution, and reproduction in any medium, provided you give appropriate credit to the original author(s) and the source, provide a link to the Creative Commons license, and indicate if changes were made. 


\section{Background}

Osteoarthritis (OA) is a debilitating disease driven by multiple inflammatory signaling pathways. There has been significant interest in the development of autologous therapies because they contain growth factors and cytokines which could inhibit numerous inflammatory signaling pathways which may drive the progression of OA (Filardo et al. 2013). Nonetheless, there are uncertainties regarding the effectiveness and associated mechanism of action of autologous therapies for specific indications (Sheth 2012). This has motivated the need for advanced autologous therapies with cellular and molecular profiles tailored to address specific diseases or injuries.

Autologous Protein Solution (APS) is a new autologous therapy under investigation for the treatment of knee OA. APS is prepared from a small volume of blood at the point-of-care. In this class of therapeutics, APS has a unique profile of concentrated white blood cells (WBCs), platelets, and plasma (O'Shaughnessey et al. 2014). WBCs are the main source of interleukin-1 receptor antagonist (IL-1ra) in the body which competitively inhibits inflammatory interleukin-1 $\beta$ (IL-1 $\beta$ ) signaling (Jordan et al. 1995). Platelets' alpha granules contain anabolic growth factors which are important in cartilage repair pathways (Sanchez et al. 2003) and synergistically act with anti-inflammatory cytokines on the nuclear factor kappa-light-chain-enhancer of activated B cells $(\mathrm{NF}-\mathrm{k} \beta$ ) pathway (Montaseri et al. 2011). Plasma contains anti-inflammatory cytokines including soluble interleukin-1 receptor antagonist-type II (sIL-1RII) (Arend et al. 1994) and soluble tumor necrosis factor receptor-type I and -type II (sTNF-RI and sTNF-RII) (Arend 2002). The ability of APS to block both IL-1 $\beta$ and tumor necrosis factor $\alpha(\mathrm{TNF} \alpha)$ signaling pathways suggests that it may have utility in the treatment of OA despite the failure of other therapies which block single inflammatory cytokines with recombinant inhibitors (Malemud 2010). The unique cellular and cytokine profile of APS has motivated the exploration of its therapeutic potential.

APS has been tested in cell culture, cartilage explant culture, and in horses with naturally occurring OA. On the cellular level, APS was cultured with macrophages in inflammatory environments, and changes in IL-8 production were measured, demonstrating the ability of APS to reduce inflammation in vitro (O'Shaughnessey et al. 2011). In a separate study, chondrocytes were challenged with recombinant human IL-1 $\beta$ or TNF $\alpha$ and reduction in matrix metalloproteinase-13 (MMP-13 or collagenase) production was measured after APS was introduced to the culture (Woodell-May et al. 2011). On the tissue level, cartilage explants were combined with APS in inflammatory environments, and APS was shown to reduce extracellular matrix degradation and stimulate chondrocyte proliferation (Matuska et al. 2013). APS has been tested in a randomized and controlled trial in horses with naturally occurring OA. Changes in lameness were evaluated using force plate analysis, veterinarian evaluations, and owner surveys (Bertone et al. 2014). Finally, the cytokine profile in APS from 105 subjects with OA was characterized (O'Shaughnessey et al. 2014). Together, these data provided evidence to support an open-label trial to evaluate intra-articular injection of APS in subjects with early to moderate knee OA.

It was hypothesized that increasing concentrations of WBC in APS would be correlated with improved WOMAC pain scores. The secondary analysis described in this manuscript identified characteristics of APS that may correlate with improved Western Ontario and McMaster Universities Osteoarthritis Index (WOMAC) scores and Outcome Measures in RheumatologyOsteoarthritis Research Society International (OMERACT-OARSI) responder status after treatment with an intra-articular injection of the APS. WOMAC pain scores were analyzed in detail because of the central role of pain when evaluating the effectiveness of potential OA therapies.

\section{Methods}

This first-in-human, open-label, single-center (St. Anna Hospital, Geldrop, Netherlands) study evaluated the safety and tolerability of a single dose of APS in subjects with OA of the knee who have failed at least one conservative therapy (NCT01773226, https://clinicaltrials.gov/ ct2/show/NCT01773226). In addition to this primary objective, the secondary objective was to explore the effect of a single injection of APS on pain, joint functionality, and stiffness. Eligibility criteria for this study are summarized in Table 1 and exclusion criteria are summarized in Table 2. A total of 11 subjects were enrolled (Institutional Review Board, Medical Ethics Review Committee Number 1304) after submitting their informed consent and the study was conducted in compliance with the Helsinki Declaration. WOMAC surveys were completed at baseline and at follow-up visits throughout the study, and two APS Kits (nSTRIDE Autologous Protein Solution (APS) Kit, Biomet, USA) were processed per patient.

To process each APS device, $60 \mathrm{ml}$ of anti-coagulated blood (55 ml blood with $5 \mathrm{ml}$ anticoagulant citrate dextrose solution, solution A (ACD-A; Citra Labs, LLC) was processed using the APS Separator to form a cell solution. The cell solution is processed using the APS Concentrator to produce APS. The production of APS requires less than $20 \mathrm{~min}$ and is performed at the pointof-care. 
Table 1 Inclusion criteria for patients in the open-label study of APS on patients with OA

Inclusion Criteria

Male or female $\geq 40$ years

Willingness and ability to comply with the study procedures and visit schedules and ability to follow verbal and written instructions

A standing radiograph of the knee showing a Kellgren grade of 2 or 3 (within 6 months prior to screening or at screening)

Frequency of knee pain on most days (>15 days) over the last month

Diagnosis of unilateral knee OA according to the American College of Rheumatology (ACR) criteria (clinical and radiological categories) based on an $x$-ray performed within 6 months prior to screening

Body mass index (BMI) $\leq 40 \mathrm{~kg} / \mathrm{m}^{2}$

On the WOMAC LK 3.1 questionnaire, the mean total score of the 5 pain subscale items together must be $\geq 2.0$ at screening and at baseline (Day 1 pre-injection)

Failed at least 1 prior conservative OA therapy (physiotherapy and/or simple analgesics)

Signed an IEC-approved ICF

Willingness to abstain from the use of topical pain therapies (e.g., NSAIDs, capsaicin, lidocaine patches, heat patches), intra-articular corticosteroids, and viscosupplementation during the study

Willingness to abstain from systemic pain medications, except for rescue medication (acetaminophen, maximum daily dose $\leq 4 \mathrm{~g}$ ); also, to abstain from rescue medication for at least $48 \mathrm{~h}$ prior to post-screening study visits

The knee was cleaned with an antiseptic, and a 19 gauge needle was advanced percutaneously into the intra-articular space under ultrasound guidance. A luerlok syringe was connected to the needle, and all of the available joint fluid was aspirated. Without removing the needle from the joint, the syringe containing the APS was attached and all APS was injected. Subjects were advised to not increase physical activity level for two weeks following the injection and to maintain a stable lifestyle throughout the duration of the study. Subjects were also instructed to withhold rescue medication (acetaminophen) for at least $48 \mathrm{~h}$ prior to each follow up visit.

WBC and cytokine concentrations (ELISA kits, R\&D Systems, Minneapolis, MN) were measured from the output of the second APS Kit as well as a separate whole blood sample. IL-1ra, sIL-1RII, IL-1 $\beta$, sTNF-RII, and TNF $\alpha$ were measured in this study due to the important and synergistic roles that IL- $1 \beta$ and TNF $\alpha$ play in the progression of OA (Goldring 2000). More information on the ELISA kits can be found in Additional file 1: Table S1. The post-injection severity and frequency of pain was assessed by the patient using the WOMAC LK 3.1 questionnaire at weeks one and two and months one, three, and six post-injection.

Missing data was handled using last observation carried forward (LOCF) principles. The OMERACT-OARSI criteria were used to determine which subjects responded to APS therapy (improvement in pain $\geq 50 \%$ and absolute
Table 2 Exclusion criteria for patients in the open-label study of APS on patients with knee OA

\section{Exclusion Criteria}

Disease Related Criteria

On day 1 (pre-injection), presence of active infection or abnormal effusion in the knee as noted by a physical examination (e.g., erythema, redness, heat, swelling)

Diagnosed with rheumatoid arthritis (RA), Reiter's syndrome, psoriatic arthritis, ankylosing spondylitis, chondromalacia, arthritis secondary to other inflammatory diseases (e.g., inflammatory bowel disease [IBD], sarcoidosis, or amyloidosis)

Arthritis of metabolic origin

Ipsilateral hip OA

Untreated traumatic injury of knee

Limited daily activity for reasons other than OA

Presence of surgical hardware or other foreign body in the knee

Prior or concomitant OA treatment-related criteria

Arthroscopy or open surgery in knee within 12 months prior to screening

Intra-articular steroid injections in any joint within 3 months prior to screening

Intra-articular HA in any joint within 6 months prior to screening

Other intra-articular therapy in any joint within 3 months prior to screening

Taking systemic steroids within 2 weeks prior to screening

Planned/anticipated surgery of the knee during the study period

Patient-related criteria:

Any clinically significant results at screening (values or findings outside of normal ranges that were deemed clinically significant by the investigator)

Active or history of malignancy other than non-melanoma skin cancer

Any serious, non-malignant, significant, acute, or chronic medical condition (e.g., diabetes, cardiopathy) or active psychiatric illness that, in the investigator's opinion, could have compromised patient safety, limited the patient's ability to complete the study, and/or compromised the objectives of the study

Skin breakdown at the knee where the injection was planned to take place

Pregnant or nursing mothers, or women likely to conceive a child and unwilling to use a reliable form of birth control for the duration of the study

History of drug or alcohol dependence, and/or positive screening results from urine drug and alcohol tests

Used any investigational drug or device within 30 days prior to screening, or 5 half-lives, whichever is longer

Used any investigational biologic within 60 days prior to screening

change $>20$ ) (Pham et al. 2003). WBC analyses were not completed on the APS of the first three subjects, and APS samples were compromised for two subjects. Therefore, these data points were censored leaving nine subjects' data for cytokine correlation and eight subjects' data for WBC correlation. Regression analyses were performed using Microsoft Excel 2010 Software. Anderson-Darling analysis 
was used to determine whether the cytokine concentrations in whole blood and APS had a normal distribution (Minitab Software, State College, PA). If the concentrations across the population had a normal distribution, per Anderson-Darling analysis, then paired t-test analyses were performed. If the concentrations were non-parametric, then Wilcoxon signed-rank analyses were performed. The level of evidence of this study was: Level IV - case series with no comparison group.

\section{Results}

Twenty-one subjects were screened and 11 subjects were enrolled including four females and seven males. One patient withdrew at six weeks and 10 completed the study. Eleven subjects were included in the data set for safety, tolerability, and performance. There were no serious adverse events (SAEs). No AEs were attributed to APS. Treatment with APS resulted in a $72.7 \%$ responder rate (improvement in pain $\geq 50 \%$ and absolute change $\geq 20$ ).

Subjects had an average age of $57.5 \pm 9.5$ years, a weight of $83.6 \pm 10.7 \mathrm{~kg}$, height of $177.1 \pm 6.7 \mathrm{~cm}$ and a body mass index (BMI) of $26.6 \pm 3.1 \mathrm{~kg} / \mathrm{M}^{2} .10$ subjects were ethnically Dutch and 1 was Indonesian. The left knee was treated in $54.5 \%$ of subjects and the right knee was treated in $45.5 \%$ of subjects.

Cytokine analysis was performed to measure device performance and to begin to characterize correlation between APS cytokine content and clinical outcomes. The APS kit produced a solution with a greater concentration of cytokines than baseline whole blood (IL1ra: $p<0.001$, paired-t test), (IL-1 $\beta: p=0.009$ Wilcoxon), (sIL-1RII: $p<0.001$, paired-t test), (sTNF-RII: $p=0.009$ Wilcoxon), and (TNF $\alpha$ : $p=0.036$ Wilcoxon) (Table 3). The average ratio of IL-1ra to IL-1 $\beta$ in APS was $8,668 \pm$ 5,600 (IL-1ra $\left.\mathrm{pg}_{\mathrm{ml}} / \mathrm{IL}-1 \beta_{\mathrm{pg} / \mathrm{ml}}\right)$ (Table 3).

The WBC concentration in whole blood and APS had significant and positive correlation $(p<0.05)$ with the concentration of IL-1ra in APS. There was no significant correlation between the concentration of WBC in whole blood or APS and IL-1 $\beta$ (Table 4). Correlation analysis between WBCs and the remaining cytokine concentrations are shown in Additional file 2: Table S2.

There was no significant correlation between any characteristic of the APS that was measured in this study and subjects' WOMAC pain scores at the injection visit. The IL-1ra:IL-1 $\beta$ ratios in whole blood and APS were significantly correlated with improved WOMAC pain scores one week (blood $p=0.01$, APS $p<0.01$ ) and six months post-injection (blood $p=0.04$, APS $p=0.04$ ) (Table 5). The WBC concentration $(p=0.03)$ and IL-1ra concentration $(p=0.03)$ in APS were both significantly correlated with improved WOMAC pain scores one week post-injection. The IL-1ra concentration in whole blood $(p=0.03)$ and sTNF-RII concentration in APS $(p=0.03)$ were significantly correlated with improved WOMAC pain scores six months post-injection. The IL$1 \beta$ concentration in APS was significantly $(p=0.045)$ correlated with diminished WOMAC pain scores six months post-injection, but at no other time points. There was no significant correlation $(p>0.05)$ between the volume of APS administered and WOMAC pain scores at the preinjection visit, one week post-injection, and six months post-injection (Table 5).

It was also of interest to determine which subjects were more likely to be responders to treatment with APS. The fraction of subjects who had an IL-1ra:IL-1 $\beta$ ratio $>1000$ in their APS or had a WBC concentration in their APS of $>30 \mathrm{k} / \mu \mathrm{l}$ had a greater probability of responding to APS therapy than the study population at every time point (Table 6).

\section{Discussion}

APS is an autologous solution with high concentrations of anti-inflammatory cytokines. The ratio of IL-1ra:IL-1 $\beta$ in whole blood and APS was significantly correlated with improved WOMAC pain scores. The subset of subjects whose IL-1ra:IL-1 $\beta$ ratio was $>1000$ in their APS had a greater probability of being a OMERACT-OARSI responder than the total study population. Also, subjects with a high concentration of WBCs in their APS demonstrated a greater probability of responding to APS therapy than the total study population. Clinical trials are ongoing to better evaluate the potential utility of these characteristics as potential diagnostic markers to aid in the identification of individuals likely to respond to the APS therapy currently under investigation. The unique cellular and cytokine profile of APS may help explain the correlations observed in this manuscript.

Table 3 Average concentrations of cytokines in whole blood and APS. (average \pm standard deviation, $n=9$ )

\begin{tabular}{|c|c|c|c|c|c|}
\hline Metric & IL-1ra & sIL-1R\| & $\mathrm{IL}-1 \beta$ & sTNF-RII & TNFa \\
\hline Whole Blood Concentration (pg/ml) & $8,029 \pm 2,818$ & $6,798 \pm 1,623$ & $1.6 \pm 0.9$ & $1,036 \pm 188$ & $0.7 \pm 1.2$ \\
\hline APS Concentration (pg/ml) & $57,511 \pm 24,272$ & $20,121 \pm 6,654$ & $20.3 \pm 34.2$ & $5,520 \pm 1,174$ & $2.6 \pm 2.9$ \\
\hline Blood Concentration Range (pg/ml) & $3,450-11,750$ & $4,622-9,050$ & $0.7-3.3$ & $891-1,563$ & $0.0-3.4$ \\
\hline APS Concentration Range (pg/ml) & $23,100-97,750$ & $11,350-28,155$ & $2.8-109.3$ & $3,934-7,556$ & $0-7.5$ \\
\hline$p$ values & $6.8 \mathrm{E}-05$ & $2.0 \mathrm{E}-05$ & 0.07 & $6.2 \mathrm{E}-07$ & 0.02 \\
\hline
\end{tabular}


Table 4 Coefficient of correlation values $\left(R^{2}\right)$ of whole blood and APS for WBC with IL-1ra and IL-1 $\beta$

\begin{tabular}{lllll}
\hline & IL-1 ra $(\mathrm{pg} / \mathrm{ml})$ & & $\mathrm{IL}-1 \beta(\mathrm{pg} / \mathrm{ml})$ & \\
\hline WBC (k/ I) & Whole Blood & APS & Whole Blood & APS \\
& $\mathbf{0 . 6 2}$ & $\mathbf{0 . 8 3}$ & 0.06 & 0.08 \\
\hline
\end{tabular}

*Bold font indicates $(p<0.05)$ and positive correlation. $(n=8)$

In a previous study in which APS was prepared from 105 subjects with OA, the IL-1ra:Il- $1 \beta$ ratio could be calculated in 98 total subjects due to sample processing errors or the concentration of IL-1 $\beta$ being below the detectable range of the ELISA assay. Of these 98 subjects, 95 had an IL-1ra:IL-1 $\beta$ ratio > 1000 (96.9 \%), which represents a conservative measurement due to excluding subjects whose IL- $1 \beta$ concentrations were very low (O'Shaughnessey et al. 2014). In this study, subjects with an IL-1ra:Il-1 $\beta$ ratio $>1000$ in their APS had a greater probability of responding to APS therapy than the total study population at every time point. Previously, two APS devices were processed from each of 50 donors, and the WBC concentrations were measured on the 100 outputs. From these outputs, 94 had a WBC concentration $>30 \mathrm{k} / \mu \mathrm{l}(94 \%)$ King and Woodell-May (2014a, b) In this study a high percentage of patients had both IL1ra:IL-1 $\beta$ ratios and WBC concentrations greater than $30 \mathrm{k} / \mu \mathrm{l}$ which is similar to our previous preclinical testing. Therefore, a high percentage of subjects may respond to APS therapy, which would be a desirable trait for any OA therapy. Future testing will be required to better characterize the relationship between the IL1ra:IL-1 $\beta$ ratio, WBC concentration, and OMERACTOARSI responder status.
Table 6 Percent of OMERACT-OARSI responders in different patient populations

\begin{tabular}{lccccc}
\hline \% Responders & Week 1 & Week 2 & Week 4 & Month 3 & Month 6 \\
\hline Total Population & 20.0 & 45.5 & 63.6 & 72.7 & 72.7 \\
IL-1 ra:IL-1 $\beta$ Ratio > 1000 & 28.6 & 71.4 & 71.4 & 100.0 & 85.7 \\
WBC > 30 k/ I & 28.6 & 71.4 & 71.4 & 100.0 & 85.7 \\
\hline
\end{tabular}

*Total population $(n=11)$, patients with APS whose IL-1 ra:IL-1 $\beta$ ratio $>1000$ $(n=7 / 9)$, and with WBC concentration in APS $>30 \mathrm{k} / \mu \mathrm{l}(n=7 / 8)$

There has been debate on the role of WBCs in autologous therapies. Some in vitro studies suggest that WBCs could promote inflammation and MMP production (Assirelli et al. 2014; Braun et al. 2014; Browning et al. 2012). However, these studies and others have used platelet-rich plasma (PRP) and tissues from unmatched donor sources (Cross et al. 2015), which could induce WBCs to elicit an immune response. Some studies have demonstrated increased concentrations of catabolic factors in WBC-containing autologous products compared to baseline blood; however, these concentrations are still less than $5 \mathrm{pg} / \mathrm{ml}$ (Sundman et al. 2011). Concentrations of catabolic factors at these levels are unlikely to have any physical consequence in $\mathrm{OA}$, as there can be approximately an order of magnitude greater concentration of IL-1 $\beta$ in synovial fluid (Schlaak et al. 1996). One potential mechanism by which WBCs may have mediated the positive effect observed in this study is through microparticles from platelets polarizing monocytes in the APS to become M2 pro-healing cells during or after delivery to the joint (Vasina et al. 2011). Also, WBCcontaining autologous products have been shown to down-regulate $N F \kappa \beta$, potentially through reducing the

Table 5 Coefficient of correlation values $\left(R^{2}\right)$ of whole blood and APS metrics with WOMAC Pain scores

\begin{tabular}{|c|c|c|c|c|}
\hline Metric & Solution & WOMAC Pain-Injection Visit & WOMAC Pain-1 Week & WOMACPain-6 Months \\
\hline \multirow[t]{2}{*}{ WBC Concentration } & Whole Blood & 0.43 & 0.42 & 0.24 \\
\hline & APS & 0.01 & 0.58 & 0.22 \\
\hline \multirow[t]{2}{*}{ IL-1 ra:IL-1 $\beta$ Ratio } & Whole Blood & 0.02 & 0.64 & 0.47 \\
\hline & APS & 0.01 & 0.37 & 0.46 \\
\hline \multirow[t]{2}{*}{ IL-1ra } & Whole Blood & 0.04 & 0.30 & 0.53 \\
\hline & APS & 0.02 & 0.52 & 0.10 \\
\hline \multirow[t]{2}{*}{ sIL-1RII } & Whole Blood & 0.19 & $<0.01$ & 0.02 \\
\hline & APS & 0.03 & 0.32 & 0.30 \\
\hline \multirow[t]{2}{*}{$\mid L-1 \beta$} & Whole Blood & 0.01 & 0.09 & 0.19 \\
\hline & APS & 0.02 & 0.04 & $\underline{0.46}$ \\
\hline \multirow[t]{2}{*}{ sTNF-RII } & Whole Blood & 0.02 & 0.16 & 0.37 \\
\hline & APS & 0.04 & 0.26 & 0.52 \\
\hline \multirow[t]{2}{*}{ TNFa } & Whole Blood & $<0.01$ & 0.20 & 0.38 \\
\hline & APS & 0.02 & 0.39 & 0.35 \\
\hline Injection Volume & APS & 0.03 & 0.01 & 0.02 \\
\hline
\end{tabular}

*Bold font represents $(p<0.05)$ and improved pain scores and italicized and underlined font represent significant and diminished pain scores with significant correlation $(n>8)$ 
expression of cyclooxygenase 2 (COX2) and inducing production of $\mathrm{NF} \kappa \beta$ inhibitor $\alpha(\mathrm{I} \kappa \beta \alpha)$ by chondrocytes (Bendinelli et al. 2010). Taken together, there are multiple mechanisms which may explain the correlation between WBC concentration in APS and improvement in WOMAC pain scores. Further investigation will be required to develop a better understanding of the mechanism(s) by which WBC concentrations may impact clinical response to the APS therapy under investigation for the treatment of OA.

The high concentrations of WBCs in APS enable it to have a high ratio of anti-inflammatory cytokines to inflammatory cytokines. Previous studies have demonstrated that WBCs are the primary source of IL-1ra in humans (Jordan et al. 1995) and in APS (King and Woodell-May 2014a, b; King and Woodell-May 2013; King and Woodell-May 2014b). In this study, the ratio of IL-1ra:IL-1 $\beta$ was significantly correlated with improved WOMAC pain scores. The balance between IL1ra and IL-1 $\beta$ has been shown to play a critical role in the homeostasis of inflammation in diverse diseases in almost every organ system (Arden 2002). IL-1ra:IL-1 $\beta$ ratios of 10-1000 have been shown to inhibit the activity of IL-1 $\beta$ in cell culture and, therefore, have been used as a benchmark for autologous therapies (Wehling et al. 2007). This manuscript provides the first clinical evidence that a IL-1ra:IL-1 $\beta$ ratio of $>1000$, in the context of APS, may predict the inhibition of inflammation in OA patients. However, this study included a small number of homogeneous patients and therefore further testing is underway to better characterize these metrics with larger numbers of subjects and more diverse populations. This observation highlights that measuring the concentration of only anti-inflammatory or only inflammatory cytokines in an autologous product may provide an incomplete picture of the processes that balance inflammation.

Although we did not observe a significant correlation between soluble inhibitors of inflammatory cytokines in APS and WOMAC pain scores, they still may have played an underlying role in mediating the activity of APS. Recombinant inhibitors of IL-1 $\beta$ have not yet proven to be effective at treating OA (Chevalier et al. 2013). Therefore, a solution with a high IL-1ra:IL-1 $\beta$ ratio alone may not be sufficient to inhibit OA pain. Although they were not measured in this study, concentrated plasma in APS has high concentrations of anabolic growth factors (O'Shaughnessey et al. 2014). Anabolic growth factors, including platelet derived growth factor (PDGF) and insulin-like growth factor-1 (IGF-1), are concentrated in APS and act in concert to decrease IL- $1 \beta$-induced NF- $\kappa \beta$ activation (Montaseri et al. 2011). The unique cytokine profile in APS may have contributed to the improved WOMAC pain scores in this study.
There were several limitations of this work. As the primary endpoint of this first-in-human study was patient safety, only a relatively small number of subjects were enrolled $(n=11)$, there was no control group, and there was a relatively homogeneous population. The sample size available was further reduced due to incomplete WBC measurements on the first three subjects and improperly stored APS samples on the first two subjects. Current trials are ongoing (NCT02138890 and NCT02262364) to collect data which can be used to further examine the relationships explored in this manuscript.

\section{Conclusions}

This study provided the first evidence that WBC concentration and the ratio of IL-1ra:IL-1 $\beta$ may correlate with improvement in WOMAC pain scores in patients with early OA. More subjects and clinical studies with control groups will be necessary to validate these measurements. These results begin to bridge the preclinical testing of APS with clinical results. Preclinical testing demonstrated that the concentrated platelets, plasma, and white blood cells in APS allow it to inhibit inflammation and reduce cartilage degradation. The results of this study suggest that WBC concentration and the IL1ra:IL-1 $\beta$ ratio are candidates for further investigation and potential development as diagnostics to predict which subjects are more likely to experience an analgesic effect from APS. Future studies will be required to confirm these findings and to determine whether APS is safe and effective for the treatment of OA.

\section{Additional files}

\section{Additional file 1: Table S1. Information on ELISA assay kits. (DOCX $13.9 \mathrm{~kb})$}

Additional file 2: Table S2. Coefficient of correlation values $\left(R^{2}\right)$ of whole blood and APS for WBC with sIL-1RII, sTNF-RII, and TNFa. (DOCX $16.2 \mathrm{~kb})$

\begin{abstract}
Abbreviations
ACD-A: anticoagulant citrate dextrose solution, solution A; APS: Autologous Protein Solution; COX2: cyclooxygenase-2; ELISA: enzyme-linked immunosorbent assay; IGF-1: insulin-like growth factor-1; IL-1: interleukin-1; IL-1ra: interleukin-1 receptor antagonist; IL-8: interleukin-8; Ik $\beta a$ : inducing production of NFK $\beta$ inhibitor alpha; LOCF: last observation carried forward; MMP-13: matrix metalloproteinase-13; NF-k : nuclear factor kappa-lightchain-enhancer of activated b cells; OA: osteoarthritis; OMERACT-

OARSI: Outcome Measures in Rheumatology- Osteoarthritis Research Society International; PDGF: platelet derived growth factor; PRP: platelet-rich plasma; SAE: serious adverse events; sIL-1RII: soluble interleukin-1 receptor antagonist receptor type II; sTNF-R: soluble tumor necrosis factor receptor; TNFa: tumor necrosis factor alpha; WBC: white blood cells; WOMAC: Western Ontario and McMaster Universities Osteoarthritis Index.
\end{abstract}

\section{Competing interests}

WK, KT, and JWM are employees of Biomet Biologics. WvdW, RVD, and HS were consultants for this study. 


\section{Authors' contributions}

WK analyzed and interpreted of the data and drafted the article. WvdW provided study materials and subjects, collected and assembled data, and provided administrative, technical, and logistic support. RVD provided study materials and subjects, collected and assembled data. HS provided study materials or subjects, collected and assembled data, analyzed and interpreted the data, provided administrative, technical, and logistic support. KT designed study, critically revised the article for important intellectual content. JWM designed study, drafted the article, critically revised the article for important intellectual content. All authors were responsible for final approval of the article.

\section{Author details}

${ }^{1}$ St. Anna Hospital, Geldrop, The Netherland. ${ }^{2}$ Biomet, 56 East Bell Drive, Warsaw, IN 46581, USA.

\section{Received: 18 November 2015 Accepted: 26 January 2016}

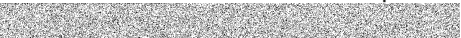

\section{References}

Arden NK (2002) The balance between IL-1 and IL-1Ra in disease. Cytokine Growth Factor Rev 13:323-340

Arend WP (2002) The mode of action of cytokine inhibitors. J Rheumatol Suppl 65:16-21

Arend WP, Malyak M, Smith MF Jr, Whisenand TD, Slack JL, Sims JE, Giri JG, Dower SK (1994) Binding of IL-1a, IL-1 3 , and IL-1 receptor antagonist by soluble IL-1 receptors and levels of soluble IL-1 receptors in synovial fluids. J Immunol 153:4766-4774

Assirelli E, Filardo G, Mariani E, Kon E, Roffi A, Vaccaro F, Marcacci M, Facchini A, Pulsatelli L (2014) Effect of two different preparations of platelet-rich plasma on synoviocytes. Knee Surg Sports Traumatol Arthrosc 23:2690-2703

Bendinelli P, Matteucci E, Dogliotti G, Corsi MM, Banfi G, Maroni P, Desiderio MA (2010) Molecular basis of anti-inflammatory action of platelet rich plasma on human chondrocytes: mechanisms of NF-kB inhibition via HGF. J Cell Physiol 225:757-766

Bertone AL, Ishihara A, Zekas LJ, Wellman ML, Lewis KB, Schwarze R, Barnaba A, Schmall ML, Kanter PM, Genovese RL (2014) Evaluation of a single intraarticular injection of autologous protein solution for treatment of osteoarthritis in horses. Am J Vet Res 75:141-151

Braun H, Kim H, Chu C, Dragoo J (2014) The effect of platelet-rich plasma formulations and blood products on human synoviocytes implications for intra-articular injury and therapy. Am J Sports Med 42:1204-1210

Browning SR, Weiser AM, Woolf N, Golish SR, Sangiovanni TP, Scuderi GJ, Carballo C, Hanna LS (2012) Platelet-rich plasma increases matrix metalloproteinases in cultures of human synovial fibroblasts. J Bone Joint Surg Am 94:e1721-e1727

Chevalier X, Eymard F, Richette P (2013) Biologic agents in osteoarthritis: hopes and disappointments. Nat Rev Rheumatol 9:400-410

Cross JA, Cole BJ, Spatny KP, Sundman E, Romeo AA, Nicholson GP, Wagner B, Fortier LA (2015) Leukocyte-reduced platelet-rich plasma normalizes matrix metabolism in torn human rotator cuff tendons. Am J Sports Med 43:2898-2906

Filardo G, Kon E, Roffi A, Di Matteo B, Merli ML, Marcacci M (2013) Platelet-rich plasma: why intra-articular? A systematic review of preclinical studies and clinical evidence on PRP for joint degeneration. Knee Surg Sports Traumatol Arthrosc 23:2459-2474

Goldring MB (2000) Osteoarthritis and cartilage: the role of cytokines. Curr Rheumatol Rep 2:459-465

Jordan M, Otterness IG, Ng R, Gessner A, Rollinghoff M, Beuscher H (1995) Neutralization of endogenous IL-6 suppresses induction of IL-1 receptor antagonist. J Immunol 154:4081-4090

King WJ and Woodell-May J (2013) Effect of leukocytes on the composition of cytokines and growth factors in two autologous preparations. Published in the Proceedings for the International Cartilage Repair Society Meeting.

King WJ and Woodell-May JE (2014) Comparison of the cellular and cytokine concentrations in the output of the autologous protein solution, orthokine, and onoccomed 2 device systems. Published in the Proceedings of the Orthopaedic Research Society Meeting

King WJ and Woodell-May JE (2014) A simple method to correlate the concentration of an anti-inflammatory cytokine with white blood cells in an autologous protein solution. Orthopaedic Research Society Spring 2014 Meeting.
Malemud CJ (2010) Anticytokine therapy for osteoarthritis: evidence to date. Drugs Aging 27:95-115

Matuska A, O'Shaughnessey KM, King WJ, Woodell-May JE (2013) Autologous solution protects bovine cartilage explants from IL-1a- and TNFa- induced cartilage degradation. J Orthop Res 31:1929-1935

Montaseri A, Busch F, Mobasheri A, Buhrmann C, Aldinger C, Rad JS, Shakibaei M (2011) IGF-1 and PDGF-bb suppress IL-1 $\beta$-induced cartilage degradation through down-regulation of NF-K $\beta$ signaling: involvement of SrC/PI-3 KNAKT pathway. PLoS One 6, e28663

O'Shaughnessey KM, Panitch A, Woodell-May JE (2011) Blood-derived antiinflammatory protein solution blocks the effect of IL-1 1 on human macrophages in vitro. Inflamm Res 60:929-936

O'Shaughnessey K, Matuska A, Hoeppner J, Farr J, Klaassen M, Kaeding C, Lattermann C, King W, Woodell-May J (2014) Autologous protein solution prepared from the blood of osteoarthritic patients contains an enhanced profile of anti-inflammatory cytokines and anabolic growth factors. J Orthop Res 32:1349-1355

Pham T, van der Heijde D, Lassere M, Altman RD, Anderson JJ, Bellamy N, Hochberg M, Simon L, Strand V, Woodworth T, Dougados M (2003) Outcome variables for osteoarthritis clinical trials: The OMERACT-OARSI set of responder criteria. J Rheumatol 30:1648-1654

Sanchez AR, Sheridan PJ, Kupp LI (2003) Is platelet-rich plasma the perfect enhancement factor? A current review. Int J Oral Maxillofac Implants 18:93-103

Schlaak JF, Pfers I, Meyer Zum Buschenfelde KH, Marker-Hermann E (1996) Different cytokine profiles in the synovial fluid of patients with osteoarthritis, rheumatoid arthritis and seronegative spondylarthropathies. Clin Exp Rheumatol 14:155-162

Sheth U (2012) Efficacy of autologous platelet-rich plasma use for orthopaedic indications: a meta-analysis. J Bone Joint Surg Am 94:298-307

Sundman EA, Cole BJ, Fortier LA (2011) Growth factor and catabolic cytokine concentrations are influenced by the cellular composition of platelet-rich plasma. Am J Sports Med 39:2135-2140

Vasina EM, Cauwenberghs S, Feijge MA, Heemskerk JW, Weber C, Koenen RR (2011) Microparticles from apoptotic platelets promote resident macrophage differentiation. Cell Death Dis 2:1-10

Wehling P, Moser C, Frisbie D, Mcllwraith CW, Kawcak CE, Krauspe R, Reinecke JA (2007) Autologous conditioned serum in the treatment of orthopedic diseases: the orthokine therapy. BioDrugs 21:323-332

Woodell-May J, Matuska A, Oyster M, Welch Z, O'Shaughnessey K, Hoeppner J (2011) Autologous protein solution inhibits MMP-13 production by IL-1 $\beta$ and TNFa-stimulated human articular chondrocytes. J Orthop Res 29:1320-1326

\section{Submit your manuscript to a SpringerOpen ${ }^{\circ}$ journal and benefit from:}

- Convenient online submission

- Rigorous peer review

- Immediate publication on acceptance

- Open access: articles freely available online

- High visibility within the field

- Retaining the copyright to your article

Submit your next manuscript at $>$ springeropen.com 